\title{
Using Time Series Analysis to forecast future RACGP OSCE capacity
}

\author{
$\underline{\text { M Buljan }}^{\mathrm{a}}$ \\ ${ }^{a}$ RMIT School of Mathematics and Geospatial Science and The Royal Australian College of General \\ Practitioners (RACGP) \\ Email: monika.buljan@racgp.org.au
}

\begin{abstract}
:
Problem:

The Australian government increased the number of first year general practice (GP) trainee places from 600 in 2010 to 1,200 in 2014 and again to 1,500 in 2015. No extra funding was provided to assess the clinical competency of the trainees to ensure they meet unsupervised Australian General Practice standards, that is, for trainees to be workforce ready.
\end{abstract}

Objective:

To forecast the timing and number of GP trainees requiring clinical assessment. The focus is the Objective Structured Clinical Examination (OSCE) at the Royal Australian College of General Practitioners (RACGP). This research will encourage a proactive approach to capacity planning and reduce potential GP workforce delays.

Participants/Data:

Historical aggregate RACGP and General Practice Education and Training (GPET) data were linked for the first time to produce time-series forecasts. Ethics approval was not required. Individuals could not be identified as only aggregate data was used.

Results:

The best forecast model from over 30 models was adjusted using GPET data. Based on two OSCEs a year, figures suggest a potential steady increase of candidates from 2014 semester 2 (2014.2) to 2016.2, with figures expected to reach about 900 and 1,100 respectively. In 2018.2, candidates are expected to peak to approximately 1,200 per semester.

For perspective, to assess 600 candidates, around 1,000 FRACGP GPs are required as examiners in a day. Current capacity is stretched to assess 800 candidates. More needs to be done to meet future expected candidates.

Conclusion:

The forecasts show how many candidates are expected to present for future OSCEs and their respective timing. This forecast can enhance current and future education and training capacity planning by planning additional exams, updating policies and continuing collaboration between organisations. Benefits of this research extend beyond the RACGP, medical workforce planning and continual professional development bodies. These forecasts could be applied in other health areas other than education. For example modelling patients, hospital resources required costs of services over time, etc.

Keywords: Forecasting, time series analysis, general practice, workforce planning 


\section{INTRODUCTION}

Medical workforce shortages are evident globally, particularly in general practice (GP) (Ettelt et al. 2008, Barber and López-Valcárcel 2010, Ono et al. 2013). With a growing population (Harrison and Britt 2011, Australian Bureau of Statistics 2013), the Australian GP shortage is one of many continuous shortages (Joyce et al. 2006, Health Workforce Australia 2012). In 2010, the Australian government committed to double GP training places to 1,200 by 2014. Trainees require clinical assessment by Australian GP colleges before they enter unsupervised practice (the workforce). Over 96\% of GP trainees in Australia are assessed by the Royal Australian College of General Practitioners (RACGP). This research explores the timing and number of GP trainees in the RACGP Objective Structured Clinical Examination (OSCE). Forecasting OSCE candidates contributes to resource planning (Hall and Viney 2008) and may reduce potential workforce delays.

The resource-heavy OSCE is based on the RACGP curriculum (The Royal Australian College of General Practitioners 2011) and Bettering the Evaluation and Care of Health (BEACH) results (Britt et al. 2012). The aggregate number of candidates who sat the OSCE was available from 2002.1 to 2013.1. This was used to forecast periods 2013.2 to 2018.1 with additional data. We assume GPs enter unsupervised practice within 12 months after passing the OSCE, thus their prompt assessment is vital. This paper is the first detailed solution to forecast RACGP OSCE candidates with General Practice Education and Training (GPET) collaboration.

Training programs and GP assessments are key factors in workforce planning. University (Del Mar and Dwyer 2006, Joyce, Stoelwinder et al. 2007) and workforce research is often limited (Anderson and Thomson 2009, Health Workforce Australia 2012). Only a subset of GPs in Australia is forecast with unrealistic or incomparable assumptions between organisations. In 2012, Health Workforce Australia (HWA) highlighted the GP shortage in Australia and provided forecast workforce figures. Their objective was to estimate Australian GP workforce which included limited detail of forecasting methodology. The purpose of this research is to plan for assessment capacity of a broader scope of general practitioners with substantial detail in the methods. Thus OSCE capacity planning forecasts are not comparable to the figures reported by HWA.

The novelty is that this research accounts for most GP trainees in Australia; it considers registrars and nonregistrars. The IMG supply is included with the assumption that patterns in IMG supply remains constant. Legislation and changes in policy will influence forecasts. With addition of new information, forecasts need to be revised. The presented forecasts were correct at the time of submission.

\section{METHODS}

Three classes of Exponential Smoothing (ES) models were applied to the RACGP data. After a comparison of the best models, GPET data was injected at the appropriate years to obtain the final forecast figures. Candidate forecasts were obtained for the next 12 semesters (6 years). The forecast computations were calculated in the statistical software, $\mathrm{R}$ (version 3.0.2).

\section{Sourcing data}

RACGP had access to historical OSCE candidates but required GPET information for accurate forecasts. Data sharing between GPET and the RACGP was limited until 2013, when a formal data exchange agreement was established. GPET first published their data in the 2012/2013 annual report (General Practice Education and Training Limited 2013).

Considerations such as registrar/ non-registrar and first-time/ repeat candidates were included in the models. Historically, around 50-70\% (Jones, Rawlin et al. 2009) of exam candidates were registrars in a given semester. Due to limited research in non-registrar trends (Hawthorne and Hamilton 2010) we assumed that historical trends would remain constant with registrar numbers increasing.

\section{Exponential Smoothing}

The ES models include parameters alpha $(\alpha)$, the data smoothing factor, beta $(\beta)$, the trend smoothing factor and gamma $(\gamma)$, the seasonality smoothing factor. The mathematical equations for ES are given below. Further theory and applications of ES and time series analysis are available in the literature (Chatfield 2013, Soyiri and Reidpath 2013).

Simple exponential smoothing

$s_{0}=x_{0}$ 
$s_{t}=\alpha x_{t-1}+(1-\alpha) s_{t-1}$ where $t>0$ and $0<\alpha<1$.

We also have $s_{t}$, the mathematical notation for the exponential smoothing algorithm at time $t$ and $x_{t}$, the historical time series observation at time $t$.

Double exponential smoothing

$s_{1}=x_{1}$

$b_{1}=x_{1}-x_{0}$

$s_{t}=\alpha x_{t}+(1-\alpha)\left(s_{t-1}+b_{t-1}\right)$

$b_{t}=\beta\left(s_{t}-s_{t-1}\right)+(1-\beta) b_{t-1}$ where $t>0,0<\alpha<1$ and $0<\beta<1$.

Here, $b_{t}$ is the best estimate of the trend at time t. All previous symbol definitions apply.

Triple exponential smoothing

$s_{0}=x_{0}$

$s_{t}=\alpha \frac{x_{t}}{c_{t-L}}+(1-\alpha)\left(s_{t-1}+b_{t-1}\right)$

$b_{t}=\beta\left(s_{t}-s_{t-1}\right)+(1-\beta) b_{t-1}$

$c_{t}=\gamma \frac{x_{t}}{s_{t}}+(1-\gamma) c_{t-L}$ where $t>0,0<\alpha<1,0<\beta<1$ and $0<\gamma<1$.

The symbol $c_{t}$ indicates the sequence of factors that correct for seasonality at time $t$ and $L$, the time length of a cycle. The definitions of all other symbols remain.

\section{Selecting parameters}

Parameters beta and gamma were set to combinations of $0,0.3,0.5$ and 0.7 . Alpha was calculated within the software. Over 35 forecast models were analysed. The models with the best visual fit and lowest sum of squared error (SSE) had their parameters refined to obtain the shortlist of 4 base models. The best simple, double (trend, no seasonality), double (seasonality, no trend) and triple model (both trend and seasonality) were selected.

\section{Adjusting the best ES model for GPET numbers}

GP registrars are eligible to sit for the OSCE about 2.5 - 3 years after they start their training program (General Practice Education and Training Limited 2014). This time lag can be added to the point estimate of any base model. First year GPET intake in excess of 600 candidates was divided by two and added to the relevant year and semester. For example, if the GPET intake was 700 in 2009, then an injection of 50 candidates would be added to the forecast point estimate in 2012.1 and 2012.2. The number of trainees who cease the training program was assumed to be negligible. Other methods of forecasting were considered, as highlighted in the discussion.

\section{RESULTS}

\section{The four base models for OSCE forecasts - (1) to (4)}

The summary statistics for the four models are summarised in Table 1. The first model used simple ES, the next two models used a trend or a seasonality factor only and the fourth model used both trend and seasonality factors:

- $\quad$ simple model

- $\quad$ double model using beta $=0.3$

- $\quad$ double model using gamma $=0.1$

- $\quad$ triple model using beta $=$ gamma $=0.05$
(1),

(2),

(3) and

The model with the smallest error seemed ideal but further exploration was required by analysing the fitted model against the observed data as well as the forecast trends. 
M. Buljan, Using Time Series Analysis to forecast future RACGP OSCE capacity

Table 1. Shortlist of Exponential Smoothing (ES) models with square root sum of squared errors

\begin{tabular}{c|cccc}
\hline Model & $\begin{array}{c}\text { Est. } \\
\text { Alpha }\end{array}$ & Beta & Gamma & sqrt(SSE) \\
\hline 1 & 0.548452 & $\mathrm{X}$ & $\mathrm{X}$ & 362.672 \\
2 & 0.686381 & 0.3 & $\mathrm{X}$ & 422.878 \\
3 & 0.675666 & $\mathrm{X}$ & 0.1 & 319.842 \\
4 & 0.760699 & 0.05 & 0.05 & 330.519 \\
\hline
\end{tabular}

\section{Fitted vs. observed graphs}

In Figure 1, the first model is underestimating the values from 2009 onwards. This implies that predictions in model (1) will probably underestimate future values. Model (2) does not estimate the historical values (early 2000 s) but comes up to the required level by 2013.1. It slightly overestimates some values. Model (3) looks very good with the peaks in the correct places but it tends to underestimate the observed values in the last few semesters. It does not capture the trend seen in the historical numbers. Model (4) has exceptional estimates from 2011 onwards and captures both the seasonality and the trend well. To further evaluate these models, the forecasts were analysed with prediction interval levels.

1) Simple

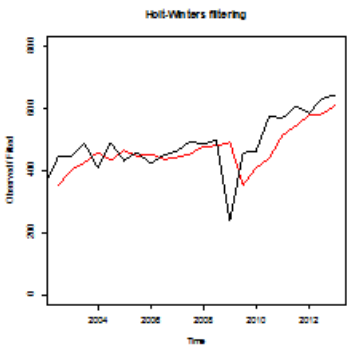

2) Double, Beta $=0.3$

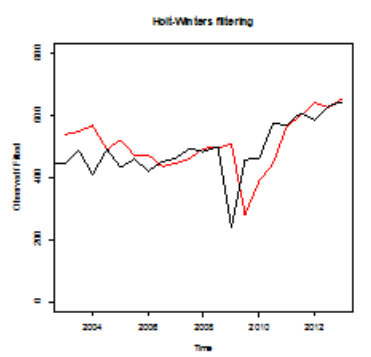

3) Double, Gamma $=0.1$

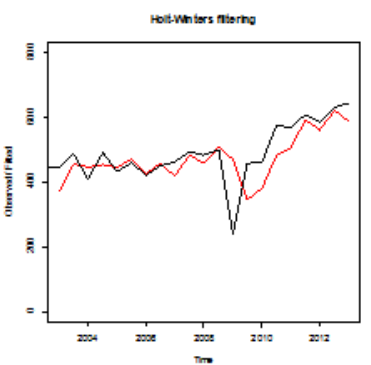

4) Triple, Beta \& Gamma = 0.05

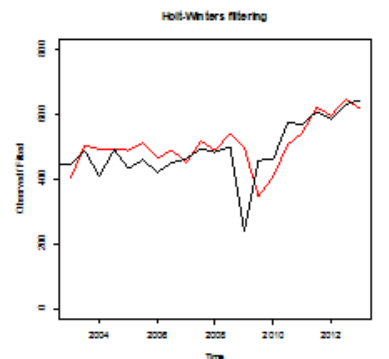

Figure 1. Visualising the fitted model (red) against the observed (black).

1) Simple

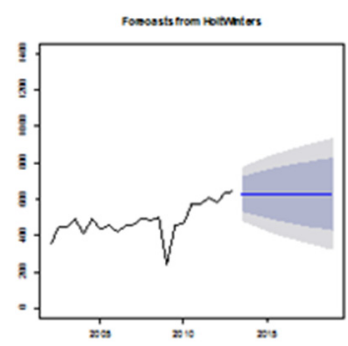

2) Double, Beta $=0.3$

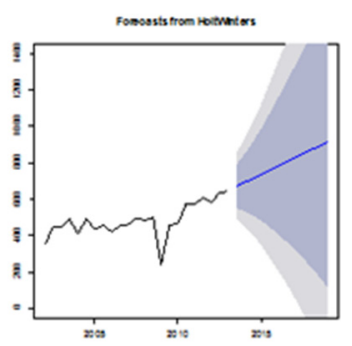

3) Double, Gamma $=0.1$

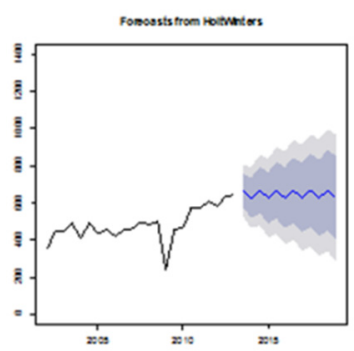

4) Triple, Beta \& Gamma $=0.05$

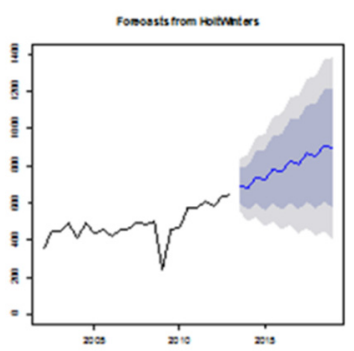

Figure 2. Visualising the forecast for the next 6 years 2013.2 to 2019.2

Forecast models (1) to (4)

The predicted models were graphed with the observed values to evaluate which model fit the data best visually. Further exploration lead to forecasting the next 12 semesters (6 years) with point estimates, $80 \%$ prediction intervals (dark grey shade) and 95\% prediction intervals (outer light grey shade), as seen in Figure 2 .

In Figure 2 shows the simple model predicts constant forecasts over the next 6 years. Prediction intervals are narrow and increase over time. The point estimate is flat with no trend or seasonality. The second model seems better. However the prediction intervals are very wide compared to the other models. The future value could fall anywhere within the prediction intervals and even outside in some circumstances. If we choose this model we have little confidence about future forecasts. 
The third model is similar to the first model except it contains seasonality. It is slightly better in predicting future values with tighter intervals, but again, we are not seeing the historical trend. The fourth model looks like a natural extension of the pattern we see historically with the point estimates. It has a narrow interval that changes for the seasonality we see between semesters. Hence Model (4) was chosen as the best model where GPET adjustments could be inserted.

\section{Adjusting Model (4): Using new GPET information to build Model (5)}

Figure 3 displays Model (4) and Model (5) on the same graph. There is a steady predicted rise of candidates per semester. OSCE figures are expected to reach close to 900 candidates in $2014.2(33.3 \%$ rise since 2012.1). By 2016.2 an OSCE would have approximately 1,100 candidates $(55.6 \%$ rise since 2012.1$)$. Numbers are expected to peak in late 2018.

\section{DISCUSSION}

The best model for forecasting future OSCE figures was Model (5). If similar resources are used in several years, one OSCE would require up to 2,000 examiners available on the same day in various Australian locations to examine 1,200 candidates. Other resources would have to double as well. The RACGP is addressing this by collaborating with organisations such as GPET and is leading projects on capacity and assessment processes. These actions will address the influx of

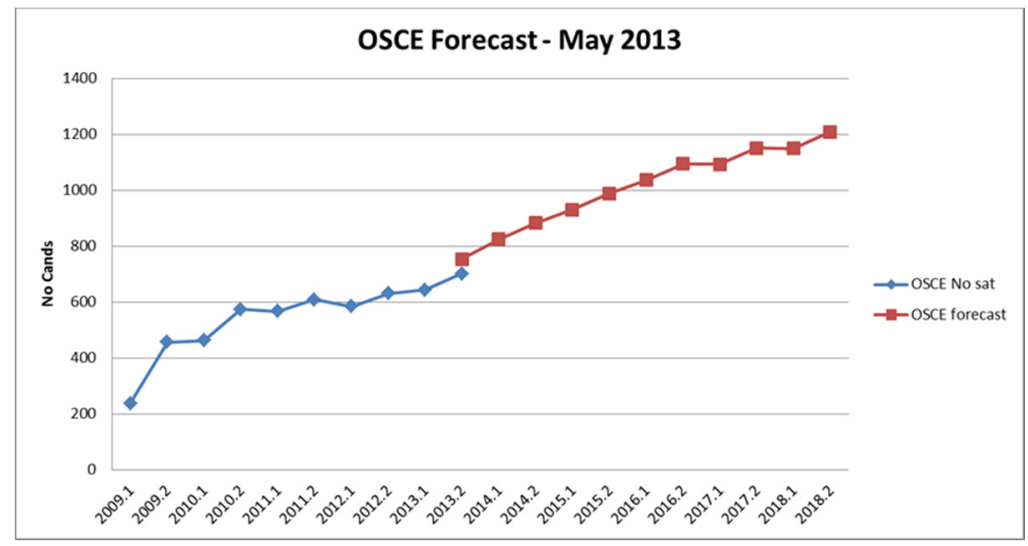

Figure 3. Final OSCE forecast model with GPET first year training intake. GPs for assessment in future.

By analysing the summary statistics and model fit of the observed versus model data, the differences were not obvious between models. Applying forecasts for the next 12 semesters (6 years) provided a better insight in model selection. Combining GPET figures with RACGP historical figures could be achieved, although some limitations were evident.

\section{Limitations}

Forecasts far in the future have very wide prediction intervals. Therefore, it is important to regularly review models as new information and data is available.

One of the limitations for all models was the change of the exam in 2009.1 which was difficult to model. However, the models can still cope with this anomaly. Another limitation included sample size of RACGP data. ES was applied to 23 data points which may be considered a "small sample size". However, no prior data could be sourced.

There are many statistical methodologies to forecast future numbers. These were peer suggestions and my consideration of them. Mathematical splines are slightly more complicated methodology to apply than ES. A simple approach was preferred for a first solution. Splines may be utilized in future forecasting. To ignore or adjust an observation (like in 2009.1) would need justification; it was preferred to keep the entire data. Since sample size was limited, two forecasts pre and post 2009 was not feasible. Forecasting separate exam cohorts was explored. Combining point estimates was possible but the confidence intervals and prediction become invalid. The combined point estimates were similar enough to the results presented in this paper, so they were omitted. Sourcing IMG trends in Australia proved to be difficult beyond the university level.

Other factors that affect OSCE numbers include:

- $\quad$ passing the Applied Knowledge Test (AKT). The AKT is a prerequisite for the OSCE. Fluctuations in AKT pass rates will affect OSCE enrolments.

- updates in RACGP policy. For example there will be an update in in 2014/15 about multiple attempts of exams. This has impact on repeat sitters that may be different to historical trends. 
- the Australian Government. They can adjust funding to training places, change the structure of training, increase or decrease the IMG workforce and change health policies. Forecasting when and if these factors will happen and their impact is difficult unless there is close collaboration and regular publications.

\section{CONCLUSIONS AND RECOMMENDATIONS}

The RACGP OSCE forecasts show how many candidates are expected to present in future clinical assessments and their respective timing. This is the first forecast of its kind that captures most GP trainees in Australia and utilizes RACGP and GPET data.

Based on two OSCEs per year, the final forecast model indicates OSCE numbers are steadily increasing over the next few years with peaks expected in late 2018 to 1,200 per semester. In 2018, the RACGP may need to administer approximately 2,300 OSCEs per year compared to about 1,300 in 2013. This has significant planning implications on resources and preparation to meet capacity needs.

The forecast methods and figures can enhance current and future education, training and workforce capacity planning. Continual professional development bodies in healthcare and general practice could use this for their own planning and expansion.

Forecasts are useful but need to be used with care. They require regular revision of assumptions. When new information or data is released the models require updating. The RACGP is continuing regular revision of forecasts so future assessment demand is met and that clinical standards in unsupervised Australian general practie is maintained.

\section{Implications for general practice}

With resources stretched to meet the current demand, more robust and/or more frequent methods of assessment must be implemented. This will meet future demands to reduce the potential risk of Australian GP workforce delays.

Collaboration between multiple organisations that educate and/or support GPs could consider sharing and publishing timely data. Intake figures, challenges and solutions on the management of increased capacity could identify relevant gaps or issues. A collaborative approach will help maintain high standard and sustainable Australian GP workforce.

\section{ACKNOWLEDGMENTS}

I would like to thank and acknowledge my employer, the RACGP; the Board of Censors, in particular, the Censor In Chief, Dr Mark Miller; for their medical insight and research endorsement. Thanks to Dr Mark Rowe and staff at GPET for their data sharing collaboration. Finally, I thank my statistical supervisory team at RMIT, Dr Lynne McArthur, Professor Panlop Zeephongsekul and Dr Margaret Wu who peer-reviewed the statistical content.

\section{REFERENCES}

Anderson, K., and Thomson, J. (2009). Vertical integration: Reducing the load on GP teachers. Australian Family Physician, 38(11), 907-910.

Australian Bureau of Statistics. (2013). Australian Demographic Statistics December quarter. Retrieved January 2014, from http://www.abs.gov.au/ausstats/abs@.nsf/mf/3101.0.

Barber, P., and López-Valcárcel, B. G. (2010). Forecasting the need for medical specialists in Spain: application of a system dynamics model. Human Resources for Health, 8, 24 . doi: http://dx.doi.org/10.1186/1478-4491-8-24.

Britt, H., Miller, G. C., Charles, J., Henderson, J., Valenti, L., Harrison, C., et al. (2012). A decade of Australian general practice activity 2002-03 to 2011-12. General practice series no. 32. Sydney University Press.

Chatfield, C. (2013). The Analysis of Time Series: An Introduction, Sixth Edition.

Del Mar, C. B., \& Dwyer, N. (2006). A radical new treatment for the sick health workforce. Medical Journal of Australia, 185(1), 32-34.

Ettelt, S., Nolte, E., Thomson, S., Mays, N., and International Healthcare Comparisons Network. (2008). Capacity planning in health care A review of the international experience. 
General Practice Education and Training Limited. (2010) GPET Welcomes New Training Places [Press release].

General Practice Education and Training Limited. (2013). Annual Report to 30 June 2013. Canberra.

General Practice Education and Training Limited. (2014). Australian General Practice Training Handbook 2015. Retrieved March, 2014, from http://www.gpet.com.au/About-Us/Policies-/Publications.

Hall, J. P., and Viney, R. C. (2008). National health reform needs strategic investment in health services research. Medical Journal of Australia, 188(1), 33-35.

Harrison, C., and Britt, H. (2011). General practice: Workforce gaps now and in 2020. Australian Family Physician, 40(1/2), 12-15.

Hawthorne, L., and Hamilton, J. (2010). International medical students and migration: the missing dimension in Australian workforce planning? Medical Journal of Australia, 193(5), 262-265.

Health Workforce Australia. (2012). 2012: Health Workforce 2025 - Volume 3 - Medical Specialties. Retrieved Feb 2013, from www.hwa.gov.au/sites/uploads/HW2025_V3_FinalReport20121109.pdf.

Jones, R., Rawlin, M., Atkinson, K., Radford, J., Au, L., \& Egglestone, D. (2009). Lessons from the past Historical trends in the RACGP examination. Australian Family Physician, 38(9), 708-710.

Joyce, C. M., McNeil, J. J., \& Stoelwinder, J. U. (2006). More doctors, but not enough: Australian medical workforce supply 2001-2012. Medical Journal of Australia, 184(9), 441-446.

Joyce, C. M., Stoelwinder, J. U., McNeil, J. J., \& Piterman, L. (2007). Riding the wave: current and emerging trends in graduates from Australian university medical schools. Medical Journal of Australia, 186(6), 309312.

Ono, T., Lafortune, G., \& Schoenstein, M. (2013). Health Workforce Planning in OECD Countries: A Review of 26 Projection Models from 18 Countries (pp. 0_1,3,6-129). Paris: Organisation for Economic Cooperation and Development (OECD).

Soyiri, I. N., \& Reidpath, D. D. (2013). An overview of health forecasting. Environmental Health and Preventive Medicine, 18(1), 1-9. doi: http://dx.doi.org/10.3134/ehtj.10.162:39-40.

The Royal Australian College of General Practitioners. (2011). Curriculum for Australian General Practice 2011. 2014, from http://curriculum.racgp.org.au. 